\title{
Fibular hemimelia
}

INSERM

\section{Source}

INSERM. (1999). Orphanet: an online rare disease and orphan drug data base. Fibular hemimelia. ORPHA:93323

Fibular hemimelia is a congenital longitudinal limb deficiency characterized by complete or partial absence of the fibula bone. 